Research Article

\title{
The Truncated Theta-EM Method for Nonlinear and Nonautonomous Hybrid Stochastic Differential Delay Equations with Poisson Jumps
}

\author{
Weifeng Wang $\mathbb{D}^{1},{ }^{1}$ Lei Yan $\mathbb{D}^{2},{ }^{2}$ Shuaibin Gao $\mathbb{D}^{1},{ }^{1}$ and Junhao Hu $\mathbb{D}^{1}$ \\ ${ }^{1}$ School of Mathematics and Statistics, South-Central University for Nationalities, Wuhan 430074, China \\ ${ }^{2}$ School of General Quality Education, Wuchang University of Technology, Wuhan 430223, China \\ Correspondence should be addressed to Junhao Hu; junhaohu74@163.com
}

Received 23 April 2021; Accepted 29 May 2021; Published 10 June 2021

Academic Editor: Shiping Wen

Copyright ( $\odot 2021$ Weifeng Wang et al. This is an open access article distributed under the Creative Commons Attribution License, which permits unrestricted use, distribution, and reproduction in any medium, provided the original work is properly cited.

In this paper, we study a class of nonlinear and nonautonomous hybrid stochastic differential delay equations with Poisson jumps (HSDDEwPJs). The convergence rate of the truncated theta-EM numerical solutions to HSDDEwPJs is investigated under given conditions. An example is shown to support our theory.

\section{Introduction}

Stochastic differential equations have been widely used in many fields and have attracted many scholars [1-3]. Sometimes, an emergency may occur in the system, and it is necessary to consider the influence of the emergency. For example, the surprising outbreak of COVID-19 has a huge impact on the world economy, especially on the stock market. Therefore, stochastic differential equations with jumps considering continuous and discontinuous random effects have been investigated to analyze these situations [4-7]. In practical applications, the parameters and forms in the stochastic systems will change when certain emergencies occur. In this case, we could use stochastic differential equations with Markovian switching to describe them [8]. In this paper, we will take the Markovian switching and jumps into consideration; i.e., we shall study hybrid stochastic differential delay equations with Poisson jumps (HSDDEwPJs).

Numerical methods have been extensively studied, due to the fact that many true solutions of plenty of stochastic differential equations could not be obtained. For example, the explicit Euler-Maruyama (EM) schemes are well known for approximating the true solutions [9]. However, when the coefficients grow superlinearly, Hutzenthaler et al. in [10] proved that, for all $p \in[1, \infty)$, the $p$ th moment of the EM approximations diverges to infinity. Therefore, many implicit methods have been proposed to approximate the solutions of the stochastic differential equations with nonlinear growing coefficients [11-13]. In addition, considering that the amount of calculations of the explicit schemes is less, some modified EM methods have been used to approximate the nonlinear stochastic differential equations [14-16]. In particular, the truncated EM method was initialized by Mao in [17] with both the drift and diffusion coefficients growing superlinearly. The convergence rate of the truncated EM method was given in [18]. Subsequently, there have been many papers discussing the truncated EM method for stochastic differential equations with superlinear coefficients [19-25]. In addition, there are many papers which consider the stability of the systems [26-30]. The truncated EM scheme for time-changed nonautonomous stochastic differential equations was shown in [31]. In [32], it was extended to the truncated theta-EM scheme on the basis of truncated EM scheme, and the strong convergence rate of the truncated theta-EM scheme for stochastic delay differential equations under local Lipschitz condition was investigated. The truncated theta-EM method will become the EM method when $\theta=0$ and degenerate to the backward EM method when $\theta=1$. Additionally, there are a few results on the numerical solutions for HSDDEwPJs. The convergence 
of EM approximation solution to the true solution in probability under some weaker conditions was proved in [33]. The EM approximate solutions converge to the true solutions for stochastic differential delay equations with Poisson jumps and Markovian switching under local Lipschitz condition [34]. The convergence of EM method for stochastic differential delay equations with Poisson jumps and Markovian switching in the sense of $L^{1}$-norm under one non-Lipschitz condition was discussed in [35]. The strong convergence between the true solutions and the numerical solutions to stochastic differential delay equations with Poisson jumps and Markovian switching was studied when the drift and diffusion coefficients are Taylor approximations [36]. To the best of our knowledge, there are few papers concerning the numerical solutions of the nonlinear and nonautonomous HSDDEwPJs. Thus, in this paper, we will give the strong convergence rate of the truncated theta-EM method for nonlinear and nonautonomous HSDDEwPJs.

This paper is organized as follows. We will introduce some necessary notations in Section 2 . The rate of convergence in $\mathscr{L}^{2}$ sense will be discussed in Section 3. Finally, in Section 4, we will give an example to illustrate that our main result could cover a large class of nonlinear and nonautonomous HSDDEwPJs.

\section{Mathematical Preliminaries}

Throughout this paper, unless otherwise specified, we will use the following notations. If $A$ is a vector or matrix, its transpose is denoted by $A^{T} . \forall x \in \mathbb{R}^{n}$, let $|x|$ denote its Euclidean norm. If $A$ is a matrix, its trace norm is denoted by $|A|=\sqrt{\operatorname{trace}\left(A^{T} A\right)}$. $A \leq 0$ and $A<0$ mean that $A$ is nonpositive and negative definite, respectively. If $a, b$ are real numbers, then $a \wedge b=\min \{a, b\}$ and $a \vee b=\max \{a, b\}$. Let $\lfloor a\rfloor$ be the largest integer which does not exceed $a$. Let $\mathbb{R}_{+}=[0,+\infty)$ and $\tau>0$. Let $\mathrm{C}\left([-\tau, 0] ; \mathbb{R}^{n}\right)$ be the family of continuous functions $v$ from $[-\tau, 0]$ to $\mathbb{R}^{n}$ with the norm $\|\nu\|=\sup _{-\tau \leq \tilde{\theta} \leq 0}|\nu(\widetilde{\theta})|$. If $H$ is a set, let $\mathbb{\square}_{H}$ denote its indicator function which means $\square_{H}(\omega)=$ 1 if $\omega \in H$ and $\nabla_{H}(\omega)=0$ if $\omega \notin H$. Let $C$ be a generic positive real constant which could be different in different cases.

Let $\left(\Omega, \mathscr{F},\left\{\mathscr{F}_{t}\right\}_{t \geq 0}, \mathbb{P}\right)$ be a complete probability space with a filtration $\left\{\mathscr{F}_{t}\right\}_{t \geq 0}$ satisfying the usual conditions (i.e., it is increasing and right continuous while $\mathscr{F}_{0}$ contains all $\mathbb{P}$-null sets). In addition, let $\mathbb{E}$ denote the probability expectation with respect to $\mathbb{P}$. For $p>0$, let $\mathrm{L}_{\mathscr{F}}^{p}\left([-\tau, 0] ; \mathbb{R}^{n}\right)$ denote the family of all $\mathscr{F}_{0}$-measurable and $C\left([-\tau, 0] ; \mathbb{R}^{n}\right)$-value random variables $\xi$ such that $\mathbb{E}\|\xi\|^{p}<\infty$. Let $B(t)=\left(B_{1}(t), \ldots, B_{m}(t)\right)^{T}$ be an $m$-dimensional Brownian motion defined on the probability space. Let $N(t)$ denote a scalar Poisson process with the compensated Poisson process $\tilde{N}(t)=N(t)-\lambda t$, where the parameter $\lambda>0$ is the jump intensity. Moreover, we assume that $B(\cdot)$ and $N(\cdot)$ are independent in this paper.

Let $r(t)(t \geq 0)$ be a right-continuous Markov chain on the probability space taking values in a finite state space $\mathbb{S}=$ $\{1,2, \ldots, N\}$ with the generator $\Gamma=\left(\gamma_{i j}\right)_{N \times N}$ given by

$$
\mathbb{P}\{r(t+\Delta)=j \mid r(t)=i\}= \begin{cases}\gamma_{i j} \Delta+o(\Delta), & \text { if } i \neq j, \\ 1+\gamma_{i i} \Delta+o(\Delta), & \text { if } i=j,\end{cases}
$$

where $\Delta>0$ and $\gamma_{i j}$ is the transition rate from $i$ to $j$ with $\gamma_{i j}>0$ if $i \neq j$, while $\gamma_{i i}=-\sum_{j \neq i} \gamma_{i j}$. We suppose that the Markov chain $r(\cdot)$ is independent of $B(\cdot)$ and $N(\cdot)$. As we know in [37], almost every sample path of $r(t)$ is a rightcontinuous step function with a finite number of simple jumps in any finite subinterval of $\mathbb{R}_{+}$. Thus, there exists a sequence of stopping times $0=\tau_{0}<\tau_{1}<\tau_{2}<\cdots<$ $\tau_{k} \longrightarrow \infty$, almost surely such that

$$
r(t)=\sum_{k=0}^{\infty} r\left(\tau_{k}\right) \rrbracket_{\left[\tau_{k}, \tau_{k+1}\right)}(t) .
$$

Hence, $r(t)$ is constant on each interval $\left[\tau_{k}, \tau_{k+1}\right)$,

$$
r(t)=r\left(\tau_{k}\right), \quad \forall t \in\left[\tau_{k}, \tau_{k+1}\right), k=0,1,2, \ldots
$$

In this paper, we consider the nonlinear and nonautonomous hybrid stochastic differential delay equations with Poisson jumps of the form

$$
\begin{aligned}
\mathrm{d} x(t)= & f(t, x(t), x(t-\tau), r(t)) \mathrm{d} t \\
& +g(t, x(t), x(t-\tau), r(t)) \mathrm{d} B(t) \\
& +h(t, x(t), x(t-\tau)) r(t)) \mathrm{d} N(t), \quad t \in[0, T],
\end{aligned}
$$

with the initial data

$$
x_{0}=\xi \in \mathrm{L}_{\mathscr{F}_{0}}^{p}\left([-\tau, 0] ; \mathbb{R}^{n}\right), \quad r(0)=r_{0} \in \mathbb{S} .
$$

Here, $f: \mathbb{R}_{+} \times \mathbb{R}^{n} \times \mathbb{R}^{n} \times \mathbb{S} \longrightarrow \mathbb{R}^{n}, g: \mathbb{R}_{+} \times \mathbb{R}^{n} \times \mathbb{R}^{n} \times$ $\mathbb{S} \longrightarrow \mathbb{R}^{n \times m}, h: \mathbb{R}_{+} \times \mathbb{R}^{n} \times \mathbb{R}^{n} \times \mathbb{S} \longrightarrow \mathbb{R}^{n}$. They are all Borel-measurable functions.

To estimate the truncated theta-EM method for (4), we need the following lemma ([8], Theorem 1.44).

Lemma 1. Given $\Delta>0$, let $r_{k}^{\Delta}=r(k \Delta)$ for $k \geq 0$. Then, $\left\{r_{k}^{\Delta}, k=0,1,2, \ldots\right\}$ is a discrete Markov chain with the onestep transition probability matrix

$$
\mathbb{P}(\Delta)=\left(\mathbb{P}_{i j}(\Delta)\right)_{N \times N}=e^{\Delta \Gamma}
$$

Then, we impose the standard hypothesis on the initial data.

Assumption 1. There exist constants $K_{1}>0$ and $\gamma \in(0,1]$ such that

$$
|\xi(t)-\xi(s)| \leq K_{1}|t-s|^{\gamma}, \quad-\tau \leq t, s \leq 0 .
$$

Since $\gamma_{i j}$ is independent of $x$, the paths of $r$ could be generated before approximating $x$. The discrete Markovian chain $\left\{r_{k}^{\Delta}, k=0,1,2, \ldots\right\}$ could be generated as follows: Compute the one-step transition probability matrix $\mathbb{P}(\Delta)$. Let $r_{0}^{\Delta}=i_{0}$, and generate a random number $\xi_{1}$ which is uniformly distributed in $[0,1]$. Define 


$$
r_{1}^{\Delta}= \begin{cases}i_{1}, & \text { if } i_{1} \in \mathbb{S}-\{N\} \text { such that } \sum_{j=1}^{i_{1}-1} \mathbb{P}_{i_{0}, j}(\Delta) \leq \xi_{1}<\sum_{j=1}^{i_{1}} \mathbb{P}_{i_{0}, j}(\Delta), \\ N, & \text { if } \sum_{j=1}^{N-1} \mathbb{P}_{i_{0}, j}(\Delta) \leq \xi_{1},\end{cases}
$$

where we set $\sum_{j=1}^{0} \mathbb{P}_{i_{0}, j}(\Delta)=0$ as usual. Then, we generate a new random number $\xi_{2}$ independently which is uniformly distributed in $[0,1]$ as well. Define

$$
r_{2}^{\Delta}=\left\{\begin{array}{l}
i_{2}, \quad \text { if } i_{2} \in \mathbb{S}-\{N\} \text { such that } \sum_{j=1}^{i_{2}-1} \mathbb{P}_{r_{1}^{\wedge}, j}(\Delta) \leq \xi_{2}<\sum_{j=1}^{i_{2}} \mathbb{P}_{r_{1}^{\Delta}, j}(\Delta), \\
N, \quad \text { if } \sum_{j=1}^{N-1} \mathbb{P}_{r_{1}^{\Delta}, j}(\Delta) \leq \xi_{2} .
\end{array}\right.
$$

Repeating this procedure, we could obtain a trajectory of $\left\{r_{k}^{\Delta}, k=1,2, \ldots\right\}$. The procedure could be applied independently to get more trajectories. After generating the discrete Markov chain $\left\{r_{k}^{\Delta}, k=0,1,2, \ldots\right\}$, we can now define the truncated theta-EM approximate solution for HSDDEwPJs (4) with the initial data (5).

In order to define the truncated theta-EM scheme, we first choose a strictly decreasing function $\varphi:(0,1] \longrightarrow$ $(0, \infty)$ such that

$$
\lim _{\Delta \longrightarrow 0} \varphi(\Delta)=\infty, \quad K_{\varphi(\Delta)} \Delta^{(1 / 4)} \leq 1, \forall \Delta \in(0,1],
$$

where $K_{\varphi(\Delta)}$ is a function that depends on $\varphi(\Delta)$. For example, we could choose

$$
\begin{aligned}
\varphi(\Delta) & =\Delta^{-\varepsilon}, \\
K_{\varphi(\Delta)} & =\varphi(\Delta),
\end{aligned}
$$

for some $\varepsilon \in(0,(1 / 8)]$.

For a given step size $\Delta \in(0,1]$, we give the definition of the truncated mapping

$$
\pi_{\Delta}(x)=(|x| \wedge \varphi(\Delta)) \frac{x}{|x|},
$$

where we let $(x /|x|)=0$ when $x=0$. The truncated functions are defined as

$$
\begin{aligned}
& f_{\Delta}(t, x, y, i)=f\left(t, \pi_{\Delta}(x), \pi_{\Delta}(y), i\right)=f\left(t,(|x| \wedge \varphi(\Delta)) \frac{x}{|x|},(|y| \wedge \varphi(\Delta)) \frac{y}{|y|}, i\right), \\
& g_{\Delta}(t, x, y, i)=g\left(t, \pi_{\Delta}(x), \pi_{\Delta}(y), i\right)=g\left(t,(|x| \wedge \varphi(\Delta)) \frac{x}{|x|},(|y| \wedge \varphi(\Delta)) \frac{y}{|y|}, i\right) .
\end{aligned}
$$

Now we give the definition of the discrete-time truncated theta-EM scheme to approximate the true solution of (4). Assume that there exist two positive integers $M$ and $M^{\prime}$ such that $\Delta=\tau / M=T / M^{\prime}$. Hence, $\Delta$ will become sufficiently small when we choose $M$ sufficiently large. Define $t_{k}=k \Delta$ for $k=-M,-M+1, \ldots, 0,1, \ldots, M^{\prime}-1$. Set $X_{\Delta}\left(t_{k}\right)=$ $\xi\left(t_{k}\right)$ for $k=-M,-M+1, \ldots, 0$ and then form

$$
\begin{aligned}
X_{\Delta}\left(t_{k+1}\right)= & X_{\Delta}\left(t_{k}\right)+\theta f_{\Delta}\left(t_{k+1}, X_{\Delta}\left(t_{k+1}\right), X_{\Delta}\left(t_{k+1-M}\right), r_{k+1}^{\Delta}\right) \Delta \\
& +(1-\theta) f_{\Delta}\left(t_{k}, X_{\Delta}\left(t_{k}\right), X_{\Delta}\left(t_{k-M}\right), r_{k}^{\Delta}\right) \Delta \\
& +g_{\Delta}\left(t_{k}, X_{\Delta}\left(t_{k}\right), X_{\Delta}\left(t_{k-M}\right), r_{k}^{\Delta}\right) \Delta B_{k} \\
& +h\left(t_{k}, X_{\Delta}\left(t_{k}\right), X_{\Delta}\left(t_{k-M}\right), r_{k}^{\Delta}\right) \Delta N_{k}
\end{aligned}
$$

for $k=0,1,2, \ldots, M^{\prime}-1$, where $\Delta B_{k}=B\left(t_{k+1}\right)-B\left(t_{k}\right)$, $\Delta N_{k}=N\left(t_{k+1}\right)-N\left(t_{k}\right)$. To form the continuous-time scheme, define

$$
\begin{aligned}
& \mu(t)=\sum_{k=0}^{M^{\prime}-1} t_{k}^{\square}\left[t_{k}, t_{k+1}\right)(t), \\
& \bar{r}(t)=\sum_{k=0}^{M^{\prime}-1} r_{k}^{\Delta} \llbracket\left[t_{k}, t_{k+1}\right)
\end{aligned}
$$

It is well known that there exist two kinds of the continuous-time step approximations. The first one is that

$$
\bar{x}_{\Delta}(t)=\sum_{k=0}^{M^{\prime}-1} X_{\Delta}\left(t_{k}\right) \mathbb{[}\left[t_{k}, t_{k+1}\right)(t) .
$$

The other one is that 


$$
\begin{aligned}
x_{\Delta}(t)= & \xi(0)-\theta f_{\Delta}\left(0, \xi(0), \xi(-\tau), r_{0}\right) \Delta \\
& +\theta f_{\Delta}\left(t, x_{\Delta}(t), x_{\Delta}(t-\tau), r(t)\right) \Delta \\
& +\int_{0}^{t} f_{\Delta}\left(\mu(s), \bar{x}_{\Delta}(s), \bar{x}_{\Delta}(s-\tau), \bar{r}(s)\right) \mathrm{d} s \\
& +\int_{0}^{t} g_{\Delta}\left(\mu(s), \bar{x}_{\Delta}(s), \bar{x}_{\Delta}(s-\tau), \bar{r}(s)\right) \mathrm{d} B(s) \\
& +\int_{0}^{t} h\left(\mu(s), \bar{x}_{\Delta}(s), \bar{x}_{\Delta}(s-\tau), \bar{r}(s)\right) \mathrm{d} N(s) .
\end{aligned}
$$

Then, we could observe that $X_{\Delta}\left(t_{k}\right)=\bar{x}_{\Delta}\left(t_{k}\right)=x_{\Delta}\left(t_{k}\right)$. Namely, they coincide at $t_{k}$. For simplicity, we write

$$
\begin{aligned}
& y_{\Delta}(t)=x_{\Delta}(t)-\theta f_{\Delta}\left(t, x_{\Delta}(t), x_{\Delta}(t-\tau), r(t)\right) \Delta, \\
& \bar{y}_{\Delta}(t)=\bar{x}_{\Delta}(t)-\theta f_{\Delta}\left(\mu(t), \bar{x}_{\Delta}(t), \bar{x}_{\Delta}(t-\tau), \bar{r}(t)\right) \Delta .
\end{aligned}
$$

\section{Convergence Rate}

To obtain the rate of convergence for the truncated theta-EM method for (4) in $\mathscr{L}^{2}$ sense, we need to impose the following assumptions on the coefficients.

Assumption 2. For any $R \geq 1$, there exists a constant $L_{R}>0$ such that

$$
\begin{aligned}
& |f(t, x, y, i)-f(t, \bar{x}, \bar{y}, i)| \mathrm{V}|g(t, x, y, i)-g(t, \bar{x}, \bar{y}, i)| \\
& \quad \leq L_{R}(|x-\bar{x}|+|y-\bar{y}|)
\end{aligned}
$$

for all $t \in[0, T], i \in \mathbb{S}$, and any $x, y, \bar{x}, \bar{y} \in \mathbb{R}^{n}$ with $|x| \vee|y| \vee|\bar{x}| \vee|\bar{y}| \leq R$.

Assumption 3. There exists a constant $K_{2}>0$ such that

$$
|h(t, x, y, i)-h(t, \bar{x}, \bar{y}, i)| \leq K_{2}(|x-\bar{x}|+|y-\bar{y}|),
$$

for all $t \in[0, T]$, any $x, y, \bar{x}, \bar{y} \in \mathbb{R}^{n}$, and $i \in \mathbb{S}$.

From Assumption 3, we could derive that there exists a constant $\bar{K}_{2}>0$ such that

$$
|h(t, x, y, i)| \leq \bar{K}_{2}(1+|x|+|y|),
$$

where $\bar{K}_{2}=3 K_{2}+\sup _{t \in[0, T], i \in \mathbb{S}}|h(t, 0,0, i)|$.

Before presenting the next assumption, we need more notations. Let $\mathcal{U}$ be the family of continuous functions $U: \mathbb{R}^{n} \times \mathbb{R}^{n} \longrightarrow \mathbb{R}_{+}$such that, for each $b>0$, there exists a positive constant $\kappa_{b}$ satisfying

$$
U(x, y) \leq \kappa_{b}|x-y|^{2},
$$

for any $x, y \in \mathbb{R}^{n}$ with $|x| \vee|y| \leq b$.

Assumption 4. There exist constants $K_{3}>0, q>2$, and $U \in \mathcal{U}$, such that

$$
\begin{aligned}
& (x-\bar{x})^{T}(f(t, x, y, i)-f(t, \bar{x}, \bar{y}, i))+\frac{q-1}{2}|g(t, x, y, i)-g(t, \bar{x}, \bar{y}, i)|^{2} \\
& \leq K_{3}\left(|x-\bar{x}|^{2}+|y-\bar{y}|^{2}\right)-U(x, \bar{x})+U(y, \bar{y}),
\end{aligned}
$$

for all $t \in[0, T]$, any $x, y, \bar{x}, \bar{y} \in \mathbb{R}^{n}$, and $i \in \mathbb{S}$.

Assumption 5. There exist constants $K_{4}>0, p>q>2$ such that

$$
x^{T} f(t, x, y, i)+\frac{p-1}{2}|g(t, x, y, i)|^{2} \leq K_{4}\left(1+|x|^{2}+|y|^{2}\right),
$$

for all $t \in[0, T]$, any $x, y \in \mathbb{R}^{n}$, and $i \in \mathbb{S}$.

Assumption 6. There exist constants $\bar{L}_{R}>0, \vartheta \in(0,1]$ and $\sigma \in(0,1]$ such that

$$
\begin{aligned}
& \left|f\left(t_{1}, x, y, i\right)-f\left(t_{2}, x, y, i\right)\right| \leq \bar{L}_{R}\left|t_{1}-t_{2}\right|^{\vartheta}, \\
& \left|g\left(t_{1}, x, y, i\right)-g\left(t_{2}, x, y, i\right)\right| \leq \bar{L}_{R}\left|t_{1}-t_{2}\right|^{\sigma},
\end{aligned}
$$

for all $t_{1}, t_{2} \in[0, T], \quad i \in \mathbb{S}$, and any $x, y \in \mathbb{R}^{n}$ with $|x| \mathrm{V}|y| \leq R$.
Assumption 7. There exist constants $K_{5}>0, \eta \in(0,1]$ such that

$$
\left|h\left(t_{1}, x, y, i\right)-h\left(t_{2}, x, y, i\right)\right| \leq K_{5}(1+|x|+|y|)\left|t_{1}-t_{2}\right|^{\eta},
$$

for all $t_{1}, t_{2} \in[0, T]$, any $x, y \in \mathbb{R}^{n}$, and $i \in \mathbb{S}$.

The boundedness of the $p$-moment of the true solution is shown in the following lemma which could be proved by using the standard method.

Lemma 2. Let Assumptions 2, 3, and 5 hold. Then, for any given initial data (5), there exists a unique solution $x(t)$ to (4) on $t \geq-\tau$. Moreover,

$$
\sup _{-\tau \leq t \leq T} \mathbb{E}|x(t)|^{q}<\infty, \quad \forall T>0 .
$$

Furthermore, we could obtain the next two lemmas in an analogous way to the proof of Lemmas 2.2 and 2.3 in [32]. 
Lemma 3. Let Assumption 2 holds. For any $\Delta \in(0,1]$ with $K_{\varphi(\Delta)} \geq 1$, we have

$$
\begin{aligned}
\left|f_{\Delta}(t, x, y, i)-f_{\Delta}(t, \bar{x}, \bar{y}, i)\right| \vee\left|g_{\Delta}(t, x, y, i)-g_{\Delta}(t, \bar{x}, \bar{y}, i)\right| & \leq 2 K_{\varphi(\Delta)}(|x-\bar{x}|+|y-\bar{y}|), \\
(x-\bar{x})^{T}\left(f_{\Delta}(t, x, y, i)-f_{\Delta}(t, \bar{x}, \bar{y}, i)\right) & \leq 5 K_{\varphi(\Delta)}^{2}\left(|x-\bar{x}|^{2}+|y-\bar{y}|^{2}\right),
\end{aligned}
$$

for all $t \in[0, T], i \in \mathbb{S}$, and any $x, y, \bar{x}, \bar{y} \in \mathbb{R}^{n}$.

From Lemma 3, we derive that

$$
\left|f_{\Delta}(t, x, y, i)\right| \vee\left|g_{\Delta}(t, x, y, i)\right| \leq 2 K_{\varphi(\Delta)}(|x|+|y|)+\sup _{t \in[0, T], i \in \mathbb{S}}[|f(t, 0,0, i)|+|g(t, 0,0, i)|] .
$$

In addition, by the monotone operator theory in [38], $5 K_{\varphi(\Delta)}^{2} \theta \Delta<1$ needs to be satisfied to ensure the existence and uniqueness of $X_{\Delta}\left(t_{k+1}\right)$ for given $X_{\Delta}\left(t_{k}\right)$. We get from (10) that $K_{\varphi(\Delta)}^{2} \Delta^{(1 / 2)} \leq 1$; thus, we need $\theta^{2} \Delta<(1 / 25)$. Moreover, to get the main result, $\theta \Delta^{(3 / 4)}<(1 / 8)$ should be satisfied in the proof of Lemma 6. Denote $\Delta^{*}=\left(1 / 25 \theta^{2}\right) \wedge\left(1 / 16 \theta^{4 / 3}\right)$. Let $\Delta \in\left(0, \Delta^{*}\right)$ and $\theta \in(0,1]$ in the rest of this paper.

Lemma 4. Let Assumption 5 hold. Then, for any $\Delta \in\left(0, \Delta^{*}\right)$ and $x, y \in \mathbb{R}^{n}$, we derive that

$$
x^{T} f_{\Delta}(t, x, y, i)+\frac{p-1}{2}\left|g_{\Delta}(t, x, y, i)\right|^{2} \leq \bar{K}_{4}\left(1+|x|^{2}+|y|^{2}\right),
$$

where $\bar{K}_{4}=3 K_{4}([1 / \varphi(1)] \vee 1)$.

Before the next lemma, define

$$
\kappa(t)=\left\lfloor\frac{t}{\Delta}\right\rfloor \Delta, \quad \forall-\tau \leq t \leq T .
$$

Lemma 5. Let Assumptions 2 and 3 hold. For any $\Delta \in\left(0, \Delta^{*}\right)$ and $t \in[0, T]$, we obtain that 


$$
\begin{aligned}
& \mathbb{E}\left(\left|y_{\Delta}(t)-\bar{y}_{\Delta}(t)\right|^{\bar{p}} \mid \mathscr{F}_{\kappa(t)}\right) \\
& \leq C \mathbb{E}\left(\left|\int_{\kappa(t)}^{t} f_{\Delta}\left(\mu(s), \bar{x}_{\Delta}(s), \bar{x}_{\Delta}(s-\tau), \bar{r}(s)\right) \mathrm{d} s\right|^{\bar{p}} \mid \mathscr{F}_{\kappa(t)}\right) \\
& +C \mathbb{E}\left(\left.\left|\int_{\kappa(t)}^{t} g_{\Delta}\left(\mu(s), \bar{x}_{\Delta}(s), \bar{x}_{\Delta}(s-\tau), \bar{r}(s)\right) \mathrm{d} B(s)\right|\right|^{\bar{p}} \mid \mathscr{F}_{\kappa(t)}\right) \\
& +C \mathbb{E}\left(\left|\int_{\kappa(t)}^{t} h\left(\mu(s), \bar{x}_{\Delta}(s), \bar{x}_{\Delta}(s-\tau), \bar{r}(s)\right) \mathrm{d} N(s)\right|^{\bar{p}} \mid \mathscr{F}_{\kappa(t)}\right) \\
& \leq C \Delta^{\bar{p}-1} \mathbb{E}\left(\int_{\kappa(t)}^{t}\left|f_{\Delta}\left(\mu(s), \bar{x}_{\Delta}(s), \bar{x}_{\Delta}(s-\tau), \bar{r}(s)\right)\right|^{\bar{p}} \mathrm{~d} s \mid \mathscr{F}_{\kappa(t)}\right) \\
& +C \Delta^{(\bar{p} / 2)-1} \mathbb{E}\left(\int_{\kappa(t)}^{t}\left|g_{\Delta}\left(\mu(s), \bar{x}_{\Delta}(s), \bar{x}_{\Delta}(s-\tau), \bar{r}(s)\right)\right|^{\bar{p}} \mathrm{~d} s \mid \mathscr{F}_{\kappa(t)}\right) \\
& +C \mathbb{E}\left(\left.\left|\int_{\kappa(t)}^{t} h\left(\mu(s), \bar{x}_{\Delta}(s), \bar{x}_{\Delta}(s-\tau), \bar{r}(s)\right) \mathrm{d} N(s)\right|\right|^{\bar{p}} \mid \mathscr{F}_{\kappa(t)}\right) \\
& \leq C \Delta^{(\bar{p} / 2)} K_{\varphi(\Delta)}^{\bar{p}}\left(1+\left|\bar{x}_{\Delta}(s)\right|^{\bar{p}}+\left|\bar{x}_{\Delta}(s-\tau)\right|^{\bar{p}}\right) \\
& +C \mathbb{E}\left(\left|\int_{\kappa(t)}^{t} h\left(\mu(s), \bar{x}_{\Delta}(s), \bar{x}_{\Delta}(s-\tau), \bar{r}(s)\right) \mathrm{d} N(s)\right|^{\bar{p}} \mid \mathscr{F}_{\kappa(t)}\right) .
\end{aligned}
$$

By the characteristic function's argument in [39], for any $\Delta \in\left(0, \Delta^{*}\right)$, we have

$$
\mathbb{E}|N(t)-N(\kappa(t))|^{\bar{p}} \leq c_{0} \Delta,
$$

$$
\begin{aligned}
& \mathbb{E}\left(\left|\int_{\kappa(t)}^{t} h\left(\mu(s), \bar{x}_{\Delta}(s), \bar{x}_{\Delta}(s-\tau), \bar{r}(s)\right) \mathrm{d} N(s)\right|^{\bar{p}} \mid \mathscr{F}_{\kappa(t)}\right) \\
& \quad \leq \mathbb{E}\left(\left|h\left(\kappa(t), x_{\Delta}(\kappa(t)), x_{\Delta}(\kappa(t-\tau)), r_{\lfloor t / \Delta\rfloor}^{\Delta}\right)(N(t)-N(\kappa(t)))\right|^{\bar{p}} \mid \mathscr{F}_{\kappa(t)}\right) \\
& \quad \leq\left|h\left(\kappa(t), x_{\Delta}(\kappa(t)), x_{\Delta}(\kappa(t-\tau)), r_{\lfloor t / \Delta\rfloor}^{\Delta}\right)\right|^{\bar{p}} \mathbb{E}|N(t)-N(\kappa(t))|^{\bar{p}} \\
& \quad \leq C \Delta\left(1+\left|\bar{x}_{\Delta}(s)\right|^{\bar{p}}+\left|\bar{x}_{\Delta}(s-\tau)\right|^{\bar{p}}\right) .
\end{aligned}
$$

Thus,

$$
\begin{aligned}
& \mathbb{E}\left(\left|y_{\Delta}(t)-\bar{y}_{\Delta}(t)\right|^{\bar{p}} \mid \mathscr{F}_{\kappa(t)}\right) \\
& \quad \leq C\left(\Delta^{(\bar{p} / 2)} K_{\varphi(\Delta)}^{\bar{p}}+\Delta\right)\left(1+\left|\bar{x}_{\Delta}(s)\right|^{\bar{p}}+\left|\bar{x}_{\Delta}(s-\tau)\right|^{\bar{p}}\right) .
\end{aligned}
$$

For $0<\bar{p}<2$, the use of Jensen's inequality yields that where $c_{0}$ is a constant independent of $\Delta$. Then, we get from Assumption 3 that 
Lemma 6. Let Assumptions 2, 3, and 5 hold; then, we have

$$
\sup _{0<\Delta<\Delta^{*}} \sup _{0 \leq t \leq T} \mathbb{E}\left|x_{\Delta}(t)\right|^{p} \leq C, \quad \forall T>0 .
$$

Proof. By (17), we get that

$$
\begin{aligned}
y_{\Delta}(t)= & y_{\Delta}(0)+\int_{0}^{t} f_{\Delta}\left(\mu(s), \bar{x}_{\Delta}(s), \bar{x}_{\Delta}(s-\tau), \bar{r}(s)\right) \mathrm{d} s \\
& +\int_{0}^{t} g_{\Delta}\left(\mu(s), \bar{x}_{\Delta}(s), \bar{x}_{\Delta}(s-\tau), \bar{r}(s)\right) \mathrm{d} B(s) \\
& +\int_{0}^{t} h\left(\mu(s), \bar{x}_{\Delta}(s), \bar{x}_{\Delta}(s-\tau), \bar{r}(s)\right) \mathrm{d} N(s),
\end{aligned}
$$

where $y_{\Delta}(0)=\xi(0)-\theta f_{\Delta}\left(0, \xi(0), \xi(-\tau), r_{0}\right) \Delta$.

Using Itô's formula, we derive that

$$
\begin{aligned}
\mathbb{E}\left|y_{\Delta}(t)\right|^{p} \leq & \mathbb{E}\left|y_{\Delta}(0)\right|^{p}+\mathbb{E} \int_{0}^{t} p\left|y_{\Delta}(s)\right|^{p-2}\left[y_{\Delta}^{T}(s) f_{\Delta}\left(\mu(s), \bar{x}_{\Delta}(s), \bar{x}_{\Delta}(s-\tau), \bar{r}(s)\right)\right. \\
& \left.+\frac{p-1}{2}\left|g_{\Delta}\left(\mu(s), \bar{x}_{\Delta}(s), \bar{x}_{\Delta}(s-\tau), \bar{r}(s)\right)\right|^{2}\right] \mathrm{d} s \\
& +\lambda \mathbb{E} \int_{0}^{t}\left(\left|x_{\Delta}(s)-h\left(\mu(s), \bar{x}_{\Delta}(s), \bar{x}_{\Delta}(s-\tau), \bar{r}(s)\right)\right|^{p}-\left|x_{\Delta}(s)\right|^{p}\right) \mathrm{d} s \\
\leq & \mathbb{E}\left|y_{\Delta}(0)\right|^{p}+\mathbb{E} \int_{0}^{t} p\left|y_{\Delta}(s)\right|^{p-2}\left[\bar{x}_{\Delta}^{T}(s) f_{\Delta}\left(\mu(s), \bar{x}_{\Delta}(s), \bar{x}_{\Delta}(s-\tau), \bar{r}(s)\right)+\frac{p-1}{2}\left|g_{\Delta}\left(\mu(s), \bar{x}_{\Delta}(s), \bar{x}_{\Delta}(s-\tau), \bar{r}(s)\right)\right|^{2}\right] \mathrm{d} s \\
& +\mathbb{E} \int_{0}^{t} p\left|y_{\Delta}(s)\right|^{p-2}\left(y_{\Delta}(s)-\bar{x}_{\Delta}(s)\right)^{T} f_{\Delta}\left(\mu(s), \bar{x}_{\Delta}(s), \bar{x}_{\Delta}(s-\tau), \bar{r}(s)\right) \mathrm{d} s \\
& +\lambda \mathbb{E} \int_{0}^{t}\left(\left|x_{\Delta}(s)-h\left(\mu(s), \bar{x}_{\Delta}(s), \bar{x}_{\Delta}(s-\tau), \bar{r}(s)\right)\right|^{p}-\left|x_{\Delta}(s)\right|^{p}\right) \mathrm{d} s=: \mathbb{E}\left|y_{\Delta}(0)\right|^{p}+A_{1}+A_{2}+A_{3} .
\end{aligned}
$$

With the help of (10), Lemma 4, and Young's inequality, we could obtain

$$
\begin{aligned}
A_{1} \leq & p \bar{K}_{4} \mathbb{E} \int_{0}^{t}\left|y_{\Delta}(s)\right|^{p-2}\left(1+\left|\bar{x}_{\Delta}(s)\right|^{2}+\left|\bar{x}_{\Delta}(s-\tau)\right|^{2}\right) \mathrm{d} s \\
\leq & p \bar{K}_{4} \mathbb{E} \int_{0}^{t}\left|x_{\Delta}(s)-\theta f_{\Delta}\left(s, x_{\Delta}(s), x_{\Delta}(s-\tau), r(s)\right) \Delta\right|^{p-2} \\
& \cdot\left(1+\left|\bar{x}_{\Delta}(s)\right|^{2}+\left|\bar{x}_{\Delta}(s-\tau)\right|^{2}\right) \mathrm{d} s \\
\leq & C \mathbb{E} \int_{0}^{t}\left(1+\left|x_{\Delta}(s)\right|^{p}+K_{\varphi(\Delta)}^{p} \Delta^{p}\left(\left|x_{\Delta}(s)\right|^{p}+\left|x_{\Delta}(s-\tau)\right|^{p}\right)\right) \\
& \left.+\left|\bar{x}_{\Delta}(s)\right|^{p}+\left|\bar{x}_{\Delta}(s-\tau)\right|^{p}\right) \mathrm{d} s \\
\leq & C\left(1+\int_{0}^{t} \sup _{0 \leq l \leq s} \mathbb{E}\left|x_{\Delta}(l)\right|^{p} \mathrm{~d} s\right) .
\end{aligned}
$$


Moreover,

$$
\begin{aligned}
A_{2} \leq & C \mathbb{E} \int_{0}^{t}\left|\bar{y}_{\Delta}(s)\right|^{p-2}\left|y_{\Delta}(s)-\bar{x}_{\Delta}(s) \| f_{\Delta}\left(\mu(s), \bar{x}_{\Delta}(s), \bar{x}_{\Delta}(s-\tau), \bar{r}(s)\right)\right| \mathrm{d} s \\
& +C \mathbb{E} \int_{0}^{t}\left|y_{\Delta}(s)-\bar{y}_{\Delta}(s)\right|^{p-2}\left|y_{\Delta}(s)-\bar{x}_{\Delta}(s) \| f_{\Delta}\left(\mu(s), \bar{x}_{\Delta}(s), \bar{x}_{\Delta}(s-\tau), \bar{r}(s)\right)\right| \mathrm{d} s \\
= & : A_{21}+A_{22} .
\end{aligned}
$$

By (10), Young's inequality, and Lemmas 3 and 5, we could show that

$$
\begin{aligned}
A_{21} \leq & C \int_{0}^{t} \mathbb{E}\left[\left|\bar{y}_{\Delta}(s)\right|^{p-2}\left|f_{\Delta}\left(\mu(s), \bar{x}_{\Delta}(s), \bar{x}_{\Delta}(s-\tau), \bar{r}(s)\right)\right|\right. \\
& \left.\cdot \mathbb{E}\left(\left|y_{\Delta}(s)-\bar{y}_{\Delta}(s)-\theta f_{\Delta}\left(\mu(s), \bar{x}_{\Delta}(s), \bar{x}_{\Delta}(s-\tau), \bar{r}(s)\right) \Delta\right| \mid \mathscr{F}_{\kappa(s)}\right)\right] \mathrm{d} s \\
\leq & C \int_{0}^{t} \mathbb{E}\left[\left|\bar{y}_{\Delta}(s)\right|^{p-2}\left|f_{\Delta}\left(\mu(s), \bar{x}_{\Delta}(s), \bar{x}_{\Delta}(s-\tau), \bar{r}(s)\right)\right|\right. \\
& \left.\cdot \mathbb{E}\left(\left|y_{\Delta}(s)-\bar{y}_{\Delta}(s)\right| \mid \mathscr{F}_{\kappa(s)}\right)\right] \mathrm{d} s \\
& +C \int_{0}^{t} \mathbb{E}\left[\left|\bar{y}_{\Delta}(s)\right|^{p-2}\left|f_{\Delta}\left(\mu(s), \bar{x}_{\Delta}(s), \bar{x}_{\Delta}(s-\tau), \bar{r}(s)\right)\right|^{2} \Delta\right] \mathrm{d} s \\
\leq & C \int_{0}^{t} \Delta^{(1 / 2)} K_{\varphi(\Delta)}^{2} \mathbb{E}\left[\left|x_{\Delta}(s)-\theta f_{\Delta}\left(s, x_{\Delta}(s), x_{\Delta}(s-\tau), r(s)\right) \Delta\right|^{p-2}\right. \\
& \left.\cdot\left(1+\left|\bar{x}_{\Delta}(s)\right|^{2}+\left|\bar{x}_{\Delta}(s-\tau)\right|^{2}\right)\right] \mathrm{d} s \\
& +C \int_{0}^{t} \Delta \mathbb{E}\left|x_{\Delta}(s)-\theta f_{\Delta}\right| s, x_{\Delta}(s), x_{\Delta}(s-\tau), r(s)|\Delta|^{p-2} \mathrm{~d} s \\
& +C \int_{0}^{t} \Delta K_{\varphi(\Delta)}^{2} \mathbb{E}\left[\left|x_{\Delta}(s)-\theta f_{\Delta}\left(s, x_{\Delta}(s), x_{\Delta}(s-\tau), r(s)\right) \Delta\right|^{p-2}\right. \\
& \left.\cdot\left(1+\left|\bar{x}_{\Delta}(s)\right|^{2}+\left|\bar{x}_{\Delta}(s-\tau)\right|^{2}\right)\right] \mathrm{d} s \\
\leq & C\left(1+\int_{0}^{t} \sup _{0 \leq l \leq s} \mathbb{E}\left|x_{\Delta}(l)\right|^{p} \mathrm{~d} s\right) . \\
& (1){ }^{t}(s)
\end{aligned}
$$

In the same way, with (10) and Lemmas 3 and 5, we have

$$
A_{22} \leq C\left(1+\int_{0}^{t} \sup _{0 \leq l \leq s} \mathbb{E}\left|x_{\Delta}(l)\right|^{p} \mathrm{~d} s\right) .
$$

By inserting (44) and (45) into (43), it follows that

$$
A_{2} \leq C\left(1+\int_{0}^{t} \sup _{0 \leq l \leq s} \mathbb{E}\left|x_{\Delta}(l)\right|^{p} \mathrm{~d} s\right) .
$$

From Assumption 3, one can see that

$$
\begin{aligned}
A_{3} & \leq\left. C \mathbb{E} \int_{0}^{t}|1+| x_{\Delta}(s)\right|^{p}+\left|\bar{x}_{\Delta}(s)\right|^{p}+\left|\bar{x}_{\Delta}(s-\tau)\right|^{p} \mid \mathrm{d} s \\
& \leq C\left(1+\int_{0}^{t} \sup _{0 \leq l \leq s} \mathbb{E}\left|x_{\Delta}(l)\right|^{p} \mathrm{~d} s\right) .
\end{aligned}
$$

Combining (41), (42), (46), and (47) together, we obtain

$$
\sup _{0 \leq l \leq t} \mathbb{E}\left|y_{\Delta}(l)\right|^{p} \leq C\left(1+\int_{0}^{t} \sup _{0 \leq l \leq s} \mathbb{E}\left|x_{\Delta}(l)\right|^{p} \mathrm{~d} s\right) \text {. }
$$

By the inequality $|u-v|^{p} \geq 2^{1-p}|u|^{p}-|v|^{p}$ and (29), we get 


$$
\begin{aligned}
\left|y_{\Delta}(t)\right|^{p} \geq & 2^{1-p}\left|x_{\Delta}(t)\right|^{p}-\theta^{p} \Delta^{p}\left|f_{\Delta}\left(t, x_{\Delta}(t), x_{\Delta}(t-\tau), r(t)\right)\right|^{p} \\
\geq & 2^{1-p}\left|x_{\Delta}(t)\right|^{p}-2^{2 p-2} \theta^{p} \Delta^{p} K_{\varphi(\Delta)}^{p}\left(\left|x_{\Delta}(t)\right|^{p}+\left|x_{\Delta}(t-\tau)\right|^{p}\right) \\
& -2^{p-1} \theta^{p} \Delta^{p} \sup _{t \in[0, T], i \in \mathbb{S}}[|f(t, 0,0, i)|+|g(t, 0,0, i)|] \\
= & \left(2^{1-p}-2^{2 p-2} \theta^{p} \Delta^{p} K_{\varphi(\Delta)}^{p}\right)\left|x_{\Delta}(t)\right|^{p}-2^{2 p-2} \theta^{p} \Delta^{p} K_{\varphi(\Delta)}^{p}\left|x_{\Delta}(t-\tau)\right|^{p} \\
& -2^{p-1} \theta^{p} \Delta^{p} \sup _{t \in[0, T], i \in \mathbb{S}}[|f(t, 0,0, i)|+|g(t, 0,0, i)|] .
\end{aligned}
$$

Note that $\Delta<\left(1 / 16 \theta^{4 / 3}\right)$; hence,

$$
\begin{aligned}
\sup _{0 \leq l \leq t} \mathbb{E}\left|x_{\Delta}(l)\right|^{p} & \leq C\left(1+\int_{0}^{t} \sup _{0 \leq l \leq s} \mathbb{E}\left|y_{\Delta}(l)\right|^{p} \mathrm{~d} s\right) \\
& \leq C\left(1+\int_{0}^{t} \sup _{0 \leq l \leq s} \mathbb{E}\left|x_{\Delta}(l)\right|^{p} \mathrm{~d} s\right) .
\end{aligned}
$$

Applying Gronwall's inequality gives the desired result.

Lemma 7. Let Assumptions 2, 3, and 5 hold. For any $\Delta \in\left(0, \Delta^{*}\right)$ and $t \in[0, T]$, we derive that

$$
\begin{aligned}
& \mathbb{E}\left|y_{\Delta}(t)-\bar{y}_{\Delta}(t)\right|^{p} \leq C\left(\Delta^{(p / 2)} K_{\varphi(\Delta)}^{p}+\Delta\right), \quad p \geq 2, \\
& \mathbb{E}\left|y_{\Delta}(t)-\bar{y}_{\Delta}(t)\right|^{p} \leq C \Delta^{(p / 2)} K_{\varphi(\Delta)}^{p}, \quad 0<p<2, \\
& \mathbb{E}\left|x_{\Delta}(t)-\bar{x}_{\Delta}(t)\right|^{p} \leq C\left(\Delta^{(p / 2)} K_{\varphi(\Delta)}^{p}+\Delta\right), \quad p \geq 2, \\
& \mathbb{E}\left|x_{\Delta}(t)-\bar{x}_{\Delta}(t)\right|^{p} \leq C \Delta^{(p / 2)} K_{\varphi(\Delta)}^{p}, \quad 0<p<2 .
\end{aligned}
$$

Hence,

$$
\begin{aligned}
\lim _{\Delta \longrightarrow 0} \mathbb{E}\left|y_{\Delta}(t)-\bar{y}_{\Delta}(t)\right|^{p} & \\
\quad & \lim _{\Delta \longrightarrow 0} \mathbb{E}\left|x_{\Delta}(t)-\bar{x}_{\Delta}(t)\right|^{p}=0, \quad \forall p>0 .
\end{aligned}
$$

Proof. By Lemmas 5 and 6, we could obtain (51) and (52). Then, the use of a similar technique in Lemma 6 gives the following when $p \geq 2$ :

$$
\mathbb{E}\left|x_{\Delta}(t)-\bar{x}_{\Delta}(t)\right|^{p} \leq C \mathbb{E}\left|y_{\Delta}(t)-\bar{y}_{\Delta}(t)\right|^{p} \leq C\left(\Delta^{(p / 2)} K_{\varphi(\Delta)}^{p}+\Delta\right) .
$$

We could get (54) by applying Jensen's inequality.

By Lemmas 2 and 6 and Chebyshev's inequality, we could get the following lemma right away.

Lemma 8. Let Assumptions 2, 3, and 5 hold. For any number $R \geq\|\xi\|$, define the stopping time

$$
\begin{aligned}
\tau_{R} & =\inf \{t \geq 0:|x(t)| \geq R\}, \\
\tau_{\Delta, R} & =\inf \left\{t \geq 0:\left|x_{\Delta}(t)\right| \geq R\right\} .
\end{aligned}
$$

Then, we obtain that

$$
\begin{gathered}
\mathbb{P}\left(\tau_{R} \leq T\right) \leq \frac{C}{R^{2}}, \\
\mathbb{P}\left(\tau_{\Delta, R} \leq T\right) \leq \frac{C}{R^{2}} .
\end{gathered}
$$

Lemma 9. Let Assumptions 1-7 hold. Let $\Delta \in\left(0, \Delta^{*}\right)$ be sufficiently small such that $\varphi(\Delta) \geq R \vee\|\xi\|$. Then, we get

$$
\mathbb{E}\left|x\left(T \wedge \rho_{\Delta, R}\right)-x_{\Delta}\left(T \wedge \rho_{\Delta, R}\right)\right|^{2} \leq C\left(\left(K_{\varphi(\Delta)}^{4} \Delta\right) \vee \Delta^{2(\gamma \wedge \vartheta \wedge \sigma \wedge \eta)}\right),
$$

where $\rho_{\Delta, R}:=\tau_{R} \wedge \tau_{\Delta, R}$.

Proof. Let $e_{\Delta}(t)=x(t)-y_{\Delta}(t) \quad$ for $\quad t \in[0, T]$ and $\Delta \in\left(0, \Delta^{*}\right)$. For simplicity, we rewrite $\rho_{\Delta, R}=\rho$. Recalling the definition of $f_{\Delta}$ and $g_{\Delta}$, we have

$$
\begin{aligned}
& f_{\Delta}\left(\mu(s), \bar{x}_{\Delta}(s), \bar{x}_{\Delta}(s-\tau), \bar{r}(s)\right)=f\left(\mu(s), \bar{x}_{\Delta}(s), \bar{x}_{\Delta}(s-\tau), \bar{r}(s)\right), \\
& g_{\Delta}\left(\mu(s), \bar{x}_{\Delta}(s), \bar{x}_{\Delta}(s-\tau), \bar{r}(s)\right)=g\left(\mu(s), \bar{x}_{\Delta}(s), \bar{x}_{\Delta}(s-\tau), \bar{r}(s)\right),
\end{aligned}
$$

for any $0 \leq s \leq t \wedge \rho$. By Itô's formula, we could show that 


$$
\begin{aligned}
& \mathbb{E}\left|e_{\Delta}(t \wedge \rho)\right|^{2} \\
& \leq \mathbb{E}\left(\theta^{2} \Delta^{2}\left|f_{\Delta}\left(0, \xi(0), \xi(-\tau), r_{0}\right)\right|^{2}\right) \\
& +\mathbb{E} \int_{0}^{t \wedge \rho}\left[2 e_{\Delta}^{T}(s)\left(f(s, x(s), x(s-\tau), r(s))-f_{\Delta}\left(\mu(s), \bar{x}_{\Delta}(s), \bar{x}_{\Delta}(s-\tau), \bar{r}(s)\right)\right)\right. \\
& \left.+\left|g(s, x(s), x(s-\tau), r(s))-g_{\Delta}\left(\mu(s), \bar{x}_{\Delta}(s), \bar{x}_{\Delta}(s-\tau), \bar{r}(s)\right)\right|^{2}\right] \mathrm{d} s \\
& +\lambda \mathbb{E} \int_{0}^{t \wedge \rho}\left(\mid e_{\Delta}(s)+h(s, x(s), x(s-\tau), r(s))\right. \\
& \left.-\left.h\left(\mu(s), \bar{x}_{\Delta}(s), \bar{x}_{\Delta}(s-\tau), \bar{r}(s)\right)\right|^{2}-\left|e_{\Delta}(s)\right|^{2}\right) \mathrm{d} s \\
& \leq C K_{\varphi(\Delta)}^{2} \Delta^{2}+\mathbb{E} \int_{0}^{t \wedge \rho}\left[2\left(x(s)-\bar{x}_{\Delta}(s)\right)^{T}\right. \\
& \cdot\left(f(s, x(s), x(s-\tau), r(s))-f\left(\mu(s), \bar{x}_{\Delta}(s), \bar{x}_{\Delta}(s-\tau), \bar{r}(s)\right)\right) \\
& \left.+\left|g(s, x(s), x(s-\tau), r(s))-g\left(\mu(s), \bar{x}_{\Delta}(s), \bar{x}_{\Delta}(s-\tau), \bar{r}(s)\right)\right|^{2}\right] \mathrm{d} s \\
& +\mathbb{E} \int_{0}^{t \wedge \rho} 2\left(\bar{x}_{\Delta}(s)-x_{\Delta}(s)\right)^{T} \\
& \cdot\left(f(s, x(s), x(s-\tau), r(s))-f\left(\mu(s), \bar{x}_{\Delta}(s), \bar{x}_{\Delta}(s-\tau), \bar{r}(s)\right)\right) \mathrm{d} s \\
& +\mathbb{E} \int_{0}^{t \wedge \rho} 2 \theta \Delta f_{\Delta}^{T}\left(s, x_{\Delta}(s), x_{\Delta}(s-\tau), r(s)\right) \\
& \cdot\left(f(s, x(s), x(s-\tau), r(s))-f\left(\mu(s), \bar{x}_{\Delta}(s), \bar{x}_{\Delta}(s-\tau), \bar{r}(s)\right)\right) \mathrm{d} s \\
& +\lambda \mathbb{E} \int_{0}^{t \wedge \rho}\left(\mid e_{\Delta}(s)+h(s, x(s), x(s-\tau), r(s))\right. \\
& \left.-\left.h\left(\mu(s), \bar{x}_{\Delta}(s), \bar{x}_{\Delta}(s-\tau), \bar{r}(s)\right)\right|^{2}-\left|e_{\Delta}(s)\right|^{2}\right) \mathrm{d} s \\
& =: C K_{\varphi(\Delta)}^{2} \Delta^{2}+B_{1}+B_{2}+B_{3}+B_{4} \text {. }
\end{aligned}
$$

Let $\bar{q} \in(2, q)$, so

$$
\begin{aligned}
\frac{1}{2}\left|g(s, x(s), x(s-\tau), r(s))-g\left(\mu(s), \bar{x}_{\Delta}(s), \bar{x}_{\Delta}(s-\tau), \bar{r}(s)\right)\right|^{2} \\
\quad \leq \frac{\bar{q}-1}{2}\left|g(s, x(s), x(s-\tau), r(s))-g\left(s, \bar{x}_{\Delta}(s), \bar{x}_{\Delta}(s-\tau), r(s)\right)\right|^{2} \\
\quad+\frac{\bar{q}-1}{2(\bar{q}-2)}\left|g\left(s, \bar{x}_{\Delta}(s), \bar{x}_{\Delta}(s-\tau), r(s)\right)-g\left(\mu(s), \bar{x}_{\Delta}(s), \bar{x}_{\Delta}(s-\tau), \bar{r}(s)\right)\right|^{2} .
\end{aligned}
$$

Then, we have 


$$
\begin{aligned}
B_{1} \leq & 2 \mathbb{E} \int_{0}^{t \wedge \rho}\left[\left(x(s)-\bar{x}_{\Delta}(s)\right)^{T}\right. \\
& \cdot\left(f(s, x(s), x(s-\tau), r(s))-f\left(s, \bar{x}_{\Delta}(s), \bar{x}_{\Delta}(s-\tau), r(s)\right)\right) \\
& \left.+\frac{\bar{q}-1}{2}\left|g(s, x(s), x(s-\tau), r(s))-g\left(s, \bar{x}_{\Delta}(s), \bar{x}_{\Delta}(s-\tau), r(s)\right)\right|^{2}\right] \mathrm{d} s \\
& +2 \mathbb{E} \int_{0}^{t \wedge \rho}\left(x(s)-\bar{x}_{\Delta}(s)\right)^{T} \cdot\left(f\left(s, \bar{x}_{\Delta}(s), \bar{x}_{\Delta}(s-\tau), r(s)\right)-f\left(\mu(s), \bar{x}_{\Delta}(s), \bar{x}_{\Delta}(s-\tau), \bar{r}(s)\right)\right) \mathrm{d} s \\
& +\mathbb{E} \int_{0}^{t \wedge \rho} \frac{\bar{q}-1}{\bar{q}-2}\left|g\left(s, \bar{x}_{\Delta}(s), \bar{x}_{\Delta}(s-\tau), r(s)\right)-g\left(\mu(s), \bar{x}_{\Delta}(s), \bar{x}_{\Delta}(s-\tau), \bar{r}(s)\right)\right|^{2} \mathrm{~d} s \\
= & B_{11}+B_{12}+B_{13} .
\end{aligned}
$$

By Assumptions 1 and 4 and Lemma 7, it follows that

We derive from Assumption 6 that

$$
\begin{aligned}
B_{11} \leq & 2 \mathbb{E} \int_{0}^{t \wedge \rho} K_{3}\left(\left|x(s)-\bar{x}_{\Delta}(s)\right|^{2}+\left|x(s-\tau)-\bar{x}_{\Delta}(s-\tau)\right|^{2}\right) \mathrm{d} s \\
& +2 \mathbb{E} \int_{0}^{t \wedge \rho}\left(-U\left(x(s), \bar{x}_{\Delta}(s)\right)+U\left(x(s-\tau), \bar{x}_{\Delta}(s-\tau)\right)\right) \mathrm{d} s \\
\leq & 4 K_{3} \mathbb{E} \int_{0}^{t \wedge \rho}\left(\left|x(s)-x_{\Delta}(s)\right|^{2}+\left|x_{\Delta}(s)-\bar{x}_{\Delta}(s)\right|^{2}\right. \\
& \left.+\left|x(s-\tau)-x_{\Delta}(s-\tau)\right|^{2}+\left|x_{\Delta}(s-\tau)-\bar{x}_{\Delta}(s-\tau)\right|^{2}\right) \mathrm{d} s \\
& +2 \int_{-\tau}^{0} U(\xi(s), \xi(\kappa(s))) \mathrm{d} s \\
\leq & C \mathbb{E} \int_{0}^{t \wedge \rho}\left|x(s)-x_{\Delta}(s)\right|^{2} \mathrm{~d} s+C \mathbb{E} \int_{0}^{T} K_{\varphi(\Delta)}^{2} \Delta \mathrm{d} s \\
& +C \int_{-\tau}^{0}|\xi(s)-\xi(\kappa(s))|^{2} \mathrm{~d} s \\
\leq & C\left(\int_{0}^{t} \mathbb{E}\left|x(s \wedge \rho)-x_{\Delta}(s \wedge \rho)\right|^{2} \mathrm{~d} s+K_{\varphi(\Delta)}^{2} \Delta+\Delta^{2 \gamma}\right) .
\end{aligned}
$$

$$
\begin{aligned}
B_{12} \leq & \mathbb{E} \int_{0}^{t \wedge \rho}\left(\left|x(s)-\bar{x}_{\Delta}(s)\right|^{2}+\left|f\left(s, \bar{x}_{\Delta}(s), \bar{x}_{\Delta}(s-\tau), r(s)\right)-f\left(\mu(s), \bar{x}_{\Delta}(s), \bar{x}_{\Delta}(s-\tau), \bar{r}(s)\right)\right|^{2}\right) \mathrm{d} s \\
\leq & 2 \mathbb{E} \int_{0}^{t \wedge \rho}\left(\left|x(s)-x_{\Delta}(s)\right|^{2}+\left|x_{\Delta}(s)-\bar{x}_{\Delta}(s)\right|^{2}\right) \mathrm{d} s \\
& +2 \mathbb{E} \int_{0}^{t \wedge \rho}\left(\left|f\left(s, \bar{x}_{\Delta}(s), \bar{x}_{\Delta}(s-\tau), r(s)\right)-f\left(\mu(s), \bar{x}_{\Delta}(s), \bar{x}_{\Delta}(s-\tau), r(s)\right)\right|^{2}\right. \\
& \left.+\left|f\left(\mu(s), \bar{x}_{\Delta}(s), \bar{x}_{\Delta}(s-\tau), r(s)\right)-f\left(\mu(s), \bar{x}_{\Delta}(s), \bar{x}_{\Delta}(s-\tau), \bar{r}(s)\right)\right|^{2}\right) \mathrm{d} s \\
\leq & C \int_{0}^{t} \mathbb{E}\left|x(s \wedge \rho)-x_{\Delta}(s \wedge \rho)\right|^{2} \mathrm{~d} s+C K_{\varphi(\Delta)}^{2} \Delta+C \Delta^{2 \vartheta} \\
& +C \mathbb{E} \int_{0}^{t \wedge \rho}\left|f\left(\mu(s), \bar{x}_{\Delta}(s), \bar{x}_{\Delta}(s-\tau), r(s)\right)-f\left(\mu(s), \bar{x}_{\Delta}(s), \bar{x}_{\Delta}(s-\tau), \bar{r}(s)\right)\right|^{2} \mathrm{~d} s .
\end{aligned}
$$

In addition, let $j$ denote the integer part of $T / \Delta$. Thus, 


$$
\begin{aligned}
& \mathbb{E} \int_{0}^{t \wedge \rho}\left|f\left(\mu(s), \bar{x}_{\Delta}(s), \bar{x}_{\Delta}(s-\tau), r(s)\right)-f\left(\mu(s), \bar{x}_{\Delta}(s), \bar{x}_{\Delta}(s-\tau), \bar{r}(s)\right)\right|^{2} \mathrm{~d} s \\
& \quad=\sum_{k=0}^{j} \mathbb{E} \int_{t_{k}}^{t_{k+1}}\left|f\left(\mu(s), \bar{x}_{\Delta}(s), \bar{x}_{\Delta}(s-\tau), r(s)\right)-f\left(\mu(s), \bar{x}_{\Delta}(s), \bar{x}_{\Delta}(s-\tau), \bar{r}(s)\right)\right|^{2} \rrbracket_{[0, t \wedge \rho]}(s) \mathrm{d} s \\
& \quad \leq 2 \sum_{k=0}^{j} \mathbb{E} \int_{t_{k}}^{t_{k+1}}\left(\left|f\left(\mu(s), \bar{x}_{\Delta}(s), \bar{x}_{\Delta}(s-\tau), r(s)\right)\right|^{2}+\left|f\left(\mu(s), \bar{x}_{\Delta}(s), \bar{x}_{\Delta}(s-\tau), \bar{r}(s)\right)\right|^{2}\right) \mathbb{q}_{[0, t \wedge \rho]}(s) \rrbracket_{\left\{r(s) \neq r\left(t_{k}\right)\right\}} \mathrm{d} s \\
& \quad \leq C \sum_{k=0}^{j} \mathbb{E} \int_{t_{k}}^{t_{k+1}} \mathbb{E}\left(\mathbb{E}\left[\left(1+K_{\varphi(\Delta)}^{2}\left(\left|\bar{x}_{\Delta}(s)\right|^{2}+\left|\bar{x}_{\Delta}(s-\tau)\right|^{2}\right)\right) \cdot \rrbracket_{\left\{r(s) \neq r\left(t_{k}\right)\right\}} \mid r\left(t_{k}\right)\right]\right) \mathrm{d} s,
\end{aligned}
$$

where we have utilized the fact that $\bar{x}_{\Delta}(s)$ and $\bar{x}_{\Delta}(s-\tau)$ are conditionally independent of $\mathbb{q}_{\left\{r(s) \neq r\left(t_{k}\right)\right\}}$ with reference to the $\sigma$-algebra generated by $r\left(t_{k}\right)$. The application of the Markov property gives

$$
\begin{aligned}
\mathbb{E}\left(\mathbb{q}_{\left\{r(s) \neq r\left(t_{k}\right)\right\}} \mid r\left(t_{k}\right)\right) & =\sum_{i \in \mathbb{S}} \mathbb{q}_{\left\{r\left(t_{k}\right)=i\right\}} \mathbb{P}\left(r(s) \neq i \mid r\left(t_{k}\right)=i\right) \\
& \leq \max _{0 \leq i \leq N}\left(-\gamma_{i i} \Delta+o(\Delta)\right) \sum_{i \in \mathbb{S}} \mathbb{q}_{\left\{r\left(t_{k}\right)=i\right\}} \\
& \leq C \Delta+o(\Delta) .
\end{aligned}
$$

From Lemma 6, it follows that

$$
\begin{aligned}
& \mathbb{E} \int_{0}^{t \wedge \rho}\left|f\left(\mu(s), \bar{x}_{\Delta}(s), \bar{x}_{\Delta}(s-\tau), r(s)\right)-f\left(\mu(s), \bar{x}_{\Delta}(s), \bar{x}_{\Delta}(s-\tau), \bar{r}(s)\right)\right|^{2} \mathrm{~d} s \\
& \quad \leq(C \Delta+o(\Delta)) \sum_{k=0}^{j} \int_{t_{k}}^{t_{k+1}}\left(1+K_{\varphi(\Delta)}^{2} \mathbb{E}\left|\bar{x}_{\Delta}(s)\right|^{2}+K_{\varphi(\Delta)}^{2} \mathbb{E}\left|\bar{x}_{\Delta}(s-\tau)\right|^{2}\right) \mathrm{d} s \\
& \quad \leq K_{\varphi(\Delta)}^{2}(C \Delta+o(\Delta)) .
\end{aligned}
$$

Inserting (68) into (65), we get

$B_{12} \leq C\left(\int_{0}^{t} \mathbb{E}\left|x(s \wedge \rho)-x_{\Delta}(s \wedge \rho)\right|^{2} \mathrm{~d} s+K_{\varphi(\Delta)}^{2} \Delta+\Delta^{2 \vartheta}+o(\Delta)\right)$.

Similar to $B_{12}$, we obtain

$$
B_{13} \leq C\left(K_{\varphi(\Delta)}^{2} \Delta+\Delta^{2 \sigma}+o(\Delta)\right) .
$$

Combining (63), (64), (69), and (70) together gives

$$
\begin{aligned}
B_{1} \leq & C\left(\int_{0}^{t} \mathbb{E}\left|x(s \wedge \rho)-x_{\Delta}(s \wedge \rho)\right|^{2} \mathrm{~d} s+K_{\varphi(\Delta)}^{2} \Delta\right. \\
& \left.+\Delta^{2 \gamma}+\Delta^{2 \vartheta}+\Delta^{2 \sigma}+o(\Delta)\right) .
\end{aligned}
$$

By Lemmas 3 and 7, we could show that

$$
\begin{aligned}
B_{2} \leq & 4 \mathbb{E} \int_{0}^{t \wedge \rho} K_{\varphi(\Delta)}\left|x_{\Delta}(s)-\bar{x}_{\Delta}(s)\right| \cdot\left(\left|x(s)-\bar{x}_{\Delta}(s)\right|+\left|x(s-\tau)-\bar{x}_{\Delta}(s-\tau)\right|\right) \mathrm{d} s \\
\leq & 2 \mathbb{E} \int_{0}^{t \wedge \rho} K_{\varphi(\Delta)}^{2}\left|x_{\Delta}(s)-\bar{x}_{\Delta}(s)\right|^{2} \mathrm{~d} s \\
& +2 \mathbb{E} \int_{0}^{t \wedge \rho}\left(\left|x(s)-\bar{x}_{\Delta}(s)\right|+\left|x(s-\tau)-\bar{x}_{\Delta}(s-\tau)\right|\right) \mathrm{d} s \\
\leq & C\left(\int_{0}^{t} \mathbb{E}\left|x(s \wedge \rho)-x_{\Delta}(s \wedge \rho)\right|^{2} \mathrm{~d} s+K_{\varphi(\Delta)}^{4} \Delta+K_{\varphi(\Delta)}^{2} \Delta+\Delta^{2 \gamma}+\Delta^{2 \vartheta}+o(\Delta)\right),
\end{aligned}
$$


where the same techniques used in the proofs of $B_{11}$ and $B_{12}$ have been applied. Similarly,

$$
\begin{aligned}
B_{3} \leq & \mathbb{E} \int_{0}^{t \wedge \rho} \theta^{2} \Delta^{2}\left|f_{\Delta}\left(s, x_{\Delta}(s), x_{\Delta}(s-\tau), r(s)\right)\right|^{2} \mathrm{~d} s \\
& +\mathbb{E} \int_{0}^{t \wedge \rho}\left|f(s, x(s), x(s-\tau), r(s))-f\left(\mu(s), \bar{x}_{\Delta}(s), \bar{x}_{\Delta}(s-\tau), \bar{r}(s)\right)\right|^{2} \mathrm{~d} s \\
\leq & C\left(K_{\varphi(\Delta)}^{2} \Delta^{2}+K_{\varphi(\Delta)}^{2} \Delta+\Delta^{2 \gamma}+\Delta^{29}+o(\Delta)\right) .
\end{aligned}
$$

By Assumptions 3 and 7, we obtain that

$$
\begin{aligned}
B_{4} \leq & \lambda \mathbb{E} \int_{0}^{t \wedge \rho}\left(\left|e_{\Delta}(s)\right|^{2}+2\left|h(s, x(s), x(s-\tau), r(s))-h\left(\mu(s), \bar{x}_{\Delta}(s), \bar{x}_{\Delta}(s-\tau), \bar{r}(s)\right)\right|^{2}\right) \mathrm{d} s \\
\leq & 2 \lambda \mathbb{E} \int_{0}^{t \wedge \rho}\left(\left|x(s)-x_{\Delta}(s)\right|^{2}+\theta^{2} \Delta^{2}\left|f_{\Delta}\left(s, x_{\Delta}(s), x_{\Delta}(s-\tau), r(s)\right)\right|^{2}\right) \mathrm{d} s \\
& +2 \lambda \mathbb{E} \int_{0}^{t \wedge \rho}\left|h(s, x(s), x(s-\tau), r(s))-h\left(s, \bar{x}_{\Delta}(s), \bar{x}_{\Delta}(s-\tau), r(s)\right)\right|^{2} \mathrm{~d} s \\
& +2 \lambda \mathbb{E} \int_{0}^{t \wedge \rho}\left|h\left(s, \bar{x}_{\Delta}(s), \bar{x}_{\Delta}(s-\tau), r(s)\right)-h\left(\mu(s), \bar{x}_{\Delta}(s), \bar{x}_{\Delta}(s-\tau), r(s)\right)\right|^{2} \mathrm{~d} s \\
& +2 \lambda \mathbb{E} \int_{0}^{t \wedge \rho}\left|h\left(\mu(s), \bar{x}_{\Delta}(s), \bar{x}_{\Delta}(s-\tau), r(s)\right)-h\left(\mu(s), \bar{x}_{\Delta}(s), \bar{x}_{\Delta}(s-\tau), \bar{r}(s)\right)\right|^{2} \mathrm{~d} s \\
\leq & C\left(\int_{0}^{t} \mathbb{E}\left|x(s \wedge \rho)-x_{\Delta}(s \wedge \rho)\right|^{2} d s+K_{\varphi(\Delta)}^{2} \Delta^{2}+K_{\varphi(\Delta)}^{2} \Delta+\Delta^{2 \gamma}+\Delta^{2 \eta}+o(\Delta)\right),
\end{aligned}
$$

where the skills of estimating $B_{1}$ have been used. Inserting (71)-(74) into (61), one can see that

$$
\begin{aligned}
\mathbb{E}\left|e_{\Delta}(t \wedge \rho)\right|^{2} \leq & C\left(\int_{0}^{t} \mathbb{E}\left|x(s \wedge \rho)-x_{\Delta}(s \wedge \rho)\right|^{2} \mathrm{~d} s+K_{\varphi(\Delta)}^{4} \Delta\right. \\
& \left.+\Delta^{2 \gamma}+\Delta^{2 \eta}+\Delta^{2 \vartheta}+\Delta^{2 \sigma}+o(\Delta)\right) .
\end{aligned}
$$

Thus,

$$
\begin{aligned}
& \mathbb{E}\left|x(t \wedge \rho)-x_{\Delta}(t \wedge \rho)\right|^{2} \mathrm{~d} s \\
& \quad \leq \mathbb{E}\left|e_{\Delta}(t \wedge \rho)\right|^{2}+\mathbb{E}\left|\theta \Delta f_{\Delta}\left(t, x_{\Delta}(t), x_{\Delta}(t-\tau), r(t)\right)\right|^{2} \\
& \quad \leq C\left(\int_{0}^{t} \mathbb{E}\left|x(s \wedge \rho)-x_{\Delta}(s \wedge \rho)\right|^{2} \mathrm{~d} s+\left(\left(K_{\varphi(\Delta)}^{4} \Delta\right) \vee \Delta^{2(\gamma \wedge \vartheta \wedge \sigma \wedge \eta)}\right)\right) .
\end{aligned}
$$

The use of Gronwall's inequality yields that

$$
\mathbb{E}\left|x(T \wedge \rho)-x_{\Delta}(T \wedge \rho)\right|^{2} \leq C\left(\left(K_{\varphi(\Delta)}^{4} \Delta\right) \vee \Delta^{2(\gamma \wedge \vartheta \wedge \sigma \wedge \eta)}\right) .
$$

The proof is completed.

Let us now give the rate of convergence in $\mathscr{L}^{2}$ sense.
Theorem 1. Let Assumptions 1-7 hold. For any sufficiently small $\Delta \in\left(0, \Delta^{*}\right)$, we assume there exists a positive constant $c^{*}$ such that

$$
\varphi(\Delta) \geq c^{*}\left(\left(K_{\varphi(\Delta)}^{4} \Delta\right) \vee \Delta^{2(\gamma \wedge \vartheta \wedge \sigma \wedge \eta)}\right)^{(-p / 2(p-2))} .
$$

Then, for any $T>0$, we have

$$
\begin{aligned}
& \mathbb{E}\left|x(T)-x_{\Delta}(T)\right|^{2} \leq C\left(\left(K_{\varphi(\Delta)}^{4} \Delta\right) \vee \Delta^{2(\gamma \wedge \vartheta \wedge \sigma \wedge \eta)}\right), \\
& \mathbb{E}\left|x(T)-\bar{x}_{\Delta}(T)\right|^{2} \leq C\left(\left(K_{\varphi(\Delta)}^{4} \Delta\right) \vee \Delta^{2(\gamma \wedge \vartheta \wedge \sigma \wedge \eta \eta)}\right) .
\end{aligned}
$$

Proof. Let $\delta>0$ be arbitrary. By Young's inequality, we obtain

$$
\begin{aligned}
u^{2} v & =\left(\delta u^{p}\right)^{(2 / p)}\left(\frac{v^{p /(p-2)}}{\delta^{2 /(p-2)}}\right)^{(p-2) / p} \\
& \leq \frac{2 \delta}{p} u^{p}+\frac{p-2}{p \delta^{2 /(p-2)}} v^{p /(p-2)}, \quad \forall u, v>0 .
\end{aligned}
$$

Therefore, 


$$
\begin{aligned}
& \mathbb{E}\left|x(T)-x_{\Delta}(T)\right|^{2} \\
&= \mathbb{E}\left(\left|x(T)-x_{\Delta}(T)\right|^{2} \square_{\{\rho>T\}}\right)+\mathbb{E}\left(\left|x(T)-x_{\Delta}(T)\right|^{2} \square_{\{\rho \leq T\}}\right) \\
& \leq \mathbb{E}\left|x(T \wedge \rho)-x_{\Delta}(T \wedge \rho)\right|^{2}+\frac{2 \delta}{p} \mathbb{E}\left|x(T)-x_{\Delta}(T)\right|^{p} \\
&+\frac{p-2}{p \delta^{2 /(p-2)}} \mathbb{P}\{\rho \leq T\} .
\end{aligned}
$$

By Lemmas 2 and 6, we get

$$
\mathbb{E}\left|x(T)-x_{\Delta}(T)\right|^{p} \leq C .
$$

From Lemma 8, it follows that

$$
\mathbb{P}\{\rho \leq T\} \leq \mathbb{P}\left\{\tau_{R} \leq T\right\}+\mathbb{P}\left\{\tau_{\Delta, R} \leq T\right\} \leq \frac{C}{R^{2}} .
$$

We can choose $\delta=\left(K_{\varphi(\Delta)}^{4} \Delta\right) \vee \Delta^{2(\gamma \wedge \vartheta \wedge \sigma \wedge \eta)} \quad$ and $R=c^{*}\left(\left(K_{\varphi(\Delta)}^{4} \Delta\right) \vee \Delta^{2(\gamma \wedge \vartheta \wedge \sigma \wedge \eta)}\right)^{(-p / 2(p-2))}$ such that

$$
\begin{aligned}
\mathbb{E}\left|x(T)-x_{\Delta}(T)\right|^{2} \leq & \mathbb{E}\left|x(T \wedge \rho)-x_{\Delta}(T \wedge \rho)\right|^{2} \\
& +C\left(\left(K_{\varphi(\Delta)}^{4} \Delta\right) \vee \Delta^{2(\gamma \wedge \vartheta \wedge \sigma \wedge \eta)}\right) .
\end{aligned}
$$

With condition (78), we obtain

$$
\varphi(\Delta) \geq c^{*}\left(\left(K_{\varphi(\Delta)}^{4} \Delta\right) \vee \Delta^{2(\gamma \wedge \vartheta \wedge \sigma \wedge \eta)}\right)^{(-p / 2(p-2))}=R
$$

From Lemma 9 and (85), we derive that

$$
\mathbb{E}\left|x(T)-x_{\Delta}(T)\right|^{2} \leq C\left(\left(K_{\varphi(\Delta)}^{4} \Delta\right) \vee \Delta^{2(\gamma \wedge \vartheta \wedge \sigma \wedge \eta)}\right) .
$$

Then, combining Lemma 7 and (79) gives (80). We complete the proof.

\section{Example}

An example is presented to illustrate our theory in this section.

Example 1. Consider the following nonlinear and nonautonomous scalar hybrid stochastic differential delay equation with Poisson jumps:

$$
\begin{aligned}
\mathrm{d} x(t)= & f(t, x(t), x(t-\tau), r(t)) \mathrm{d} t \\
& +g(t, x(t), x(t-\tau), r(t)) \mathrm{d} B(t) \\
& +h(t, x(t), x(t-\tau), r(t)) \mathrm{d} N(t),
\end{aligned}
$$

with the initial value $x_{0}$ which satisfies Assumption 1. $B(t)$ is a scalar Brownian motion, and $N(t)$ is a scalar Poisson process with intensity $\lambda=0.4$. Furthermore, $r(t)$ is a Markovian chain defined on the state space $\mathbb{S}=\{1,2\}$ with generator

$$
\Gamma=\left(\begin{array}{cc}
-2 & 2 \\
1 & -1
\end{array}\right)
$$

Moreover, for any $t \in[0,2]$ and $x, y \in \mathbb{R}$, let

$$
\begin{aligned}
& f(t, x, y, i)= \begin{cases}-5 x^{3}+3 x \sin t+\frac{1}{8}(t(2-t))^{(1 / 3)}|y|^{(5 / 4)}, & \text { if } i=1, \\
-20 x^{5}+6 x \cos t+\frac{1}{8}(t(2-t))^{(1 / 4)}|y|^{(5 / 4)}, & \text { if } i=2,\end{cases} \\
& g(t, x, y, i)= \begin{cases}\frac{1}{2}(t(2-t))^{(1 / 3)}|x|^{(3 / 2)}, & \text { if } i=1, \\
(t(2-t))^{(1 / 4)}|x|^{(5 / 2)}, & \text { if } i=2,\end{cases} \\
& h(t, x, y, i)= \begin{cases}(t(2-t))^{(1 / 5)} x, & \text { if } i=1, \\
(t(2-t))^{(1 / 5)} y, & \text { if } i=2 .\end{cases}
\end{aligned}
$$

Obviously, Assumptions 2 and 3 hold with $L_{R}=20 R^{4}, K_{2}=1$. Moreover, when $i=1$, 


$$
\begin{aligned}
& (x-\bar{x})^{T}(f(t, x, y, 1)-f(t, \bar{x}, \bar{y}, 1)) \\
& \quad \leq(x-\bar{x})^{2}-5\left(x^{2}+x \bar{x}+\bar{x}^{2}\right)+3(x-\bar{x})^{2}+\frac{1}{8}(x-\bar{x})\left(|y|^{(5 / 4)}-|\bar{y}|^{(5 / 4)}\right) \\
& \quad \leq-\frac{5}{2}(x-\bar{x})^{2}\left(x^{2}+\bar{x}^{2}\right)+4(x-\bar{x})^{2}+\frac{25}{256}|y-\bar{y}|^{2}\left(|y|^{(1 / 4)}-|\bar{y}|^{(1 / 4)}\right)^{2} \\
& \quad \leq-\frac{5}{2}(x-\bar{x})^{2}\left(|x|^{2}+|\bar{x}|^{2}\right)+4|x-\bar{x}|^{2}+\frac{25}{64}|y-\bar{y}|^{2}+\frac{25}{128}|y-\bar{y}|^{2}\left(|y|^{2}+|\bar{y}|^{2}\right)
\end{aligned}
$$

Let $q=3$. In a similar way, we have

$$
\begin{aligned}
& \frac{q-1}{2}|g(t, x, y, 1)-g(t, \bar{x}, \bar{y}, 1)|^{2} \\
& \leq\left.\frac{1}{2}|| x\right|^{(3 / 2)}-\left.|\bar{x}|^{(3 / 2)}\right|^{2} \\
& \leq \frac{9}{8}|x-\bar{x}|^{2}\left(|x|^{(1 / 2)}+|\bar{x}|^{(1 / 2)}\right)^{2} \\
& \leq \frac{9}{2}|x-\bar{x}|^{2}+\frac{9}{4}|x-\bar{x}|^{2}\left(|x|^{2}+|\bar{x}|^{2}\right) .
\end{aligned}
$$

$$
(x-\bar{x})^{T}(f(t, x, y, 1)-f(t, \bar{x}, \bar{y}, 1))
$$

$$
\begin{aligned}
& +\frac{q-1}{2}|g(t, x, y, 1)-g(t, \bar{x}, \bar{y}, 1)|^{2} \\
\leq & 9\left(|x-\bar{x}|^{2}+|y-\bar{y}|^{2}\right)-\frac{1}{4}|x-\bar{x}|^{2}\left(|x|^{2}+|\bar{x}|^{2}\right) \\
& +\frac{1}{4}|y-\bar{y}|^{2}\left(|y|^{2}+|\bar{y}|^{2}\right) .
\end{aligned}
$$

Similarly, for $i=2$, we have

Thus,

$$
\begin{aligned}
(x- & \bar{x})^{T}(f(t, x, y, 2)-f(t, \bar{x}, \bar{y}, 2)) \\
\leq & (x-\bar{x})^{2}\left(-20\left(x^{4}+x^{3} \bar{x}+x^{2} \bar{x}^{2}+x \bar{x}^{3}+\bar{x}^{4}\right)\right)+6(x-\bar{x})^{2} \\
& +\frac{1}{8}(x-\bar{x})\left(|y|^{(5 / 4)}-|\bar{y}|^{(5 / 4)}\right) \\
\leq & -10(x-\bar{x})^{2}\left(|x|^{4}+|\bar{x}|^{4}\right)+7|x-\bar{x}|^{2}+\frac{25}{256}|y-\bar{y}|^{2}\left(|y|^{(1 / 4)}+|\bar{y}|^{(1 / 4)}\right)^{2} \\
\leq & -10(x-\bar{x})^{2}\left(|x|^{4}+|\bar{x}|^{4}\right)+7|x-\bar{x}|^{2}+\frac{25}{64}|y-\bar{y}|^{2}+\frac{25}{128}|y-\bar{y}|^{2}\left(|y|^{4}+|\bar{y}|^{4}\right) \\
& \quad \frac{q-1}{2}|g(t, x, y, 2)-g(t, \bar{x}, \bar{y}, 2)|^{2} \\
\leq & \left.\frac{1}{2}|| x\right|^{(5 / 2)}-\left.|\bar{x}|^{(5 / 2)}\right|^{2} \\
\leq & \frac{25}{8}|x-\bar{x}|^{2}\left(|x|^{(1 / 2)}+|\bar{x}|^{(1 / 2)}\right)^{2} \\
\leq & \frac{25}{2}|x-\bar{x}|^{2}+\frac{25}{4}|x-\bar{x}|^{2}\left(|x|^{4}+|\bar{x}|^{4}\right) .
\end{aligned}
$$

Therefore,

$$
\begin{aligned}
& (x-\bar{x})^{T}(f(t, x, y, 2)-f(t, \bar{x}, \bar{y}, 2))+\frac{q-1}{2}|g(t, x, y, 2)-g(t, \bar{x}, \bar{y}, 2)|^{2} \\
& \quad \leq 20\left(|x-\bar{x}|^{2}+|y-\bar{y}|^{2}\right)-\frac{75}{4}|x-\bar{x}|^{2}\left(|x|^{4}+|\bar{x}|^{4}\right)+\frac{75}{4}|y-\bar{y}|^{2}\left(|y|^{4}+|\bar{y}|^{4}\right) .
\end{aligned}
$$


Hence, Assumption 4 is satisfied for any $i \in \mathbb{S}$. For Assumption 5 , by letting $p=4$, we obtain that

$$
\begin{array}{rl}
x^{T} & f(t, x, y, 1)+\frac{p-1}{2}|g(t, x, y, 1)|^{2} \\
& \leq-5 x^{4}+3 x^{2}+\frac{1}{8} x|y|^{(5 / 4)}+\frac{3}{8}|x|^{3} \leq C\left(1+|x|^{2}+|y|^{2}\right), \\
& \cdot x^{T} f(t, x, y, 2)+\frac{p-1}{2}|g(t, x, y, 2)|^{2} \\
& \leq-20 x^{6}+6 x^{2}+\frac{1}{8} x|y|^{(5 / 4)}+\frac{3}{2}|x|^{5} \leq C\left(1+|x|^{2}+|y|^{2}\right) .
\end{array}
$$

Thus, Assumption 5 is satisfied. Then, it is easy to see that Assumptions 6 and 7 hold with $\vartheta=\sigma=(1 / 4), \eta=(1 / 5)$.

For any $\varepsilon \in(0,(1 / 4)]$, define $\varphi(\Delta)=\sqrt{[4]}(1 / 20) \Delta^{-(\varepsilon / 4)}$. Therefore, when $K_{\varphi(\Delta)}=20(\varphi(\Delta))^{4}$, we have $K_{\varphi(\Delta)} \Delta^{(1 / 4)}=$ $\Delta^{(1 / 4)-\varepsilon} \leq 1$ and $\lim _{\Delta \longrightarrow 0} \varphi(\Delta)=\infty$. Choose $p=4, \varepsilon=$ $(33 / 136), c^{*}=\sqrt{[4]}(1 / 20)$; then, we derive that

$$
\sqrt[4]{\frac{1}{20}}\left(K_{\varphi(\Delta)}^{4} \Delta\right)^{(-p / 2(p-2))}=\sqrt[4]{\frac{1}{20}} \Delta^{4 \varepsilon-1} \leq \sqrt[4]{\frac{1}{20}} \Delta^{-(\varepsilon / 4)}=\varphi(\Delta) .
$$

By Theorem 1, we could get that

$$
\mathbb{E}\left|x(T)-x_{\Delta}(T)\right|^{2} \leq C \Delta^{(1-4 \varepsilon) \wedge 2 \gamma},
$$

where $\gamma \in(0,1]$ is defined in Assumption 1. Thus, the convergence rate of the truncated theta-EM method for (88) is $(1-4 \varepsilon) / 2 \wedge \gamma$. This example shows that our main result could cover a large class of nonlinear and nonautonomous HSDDEwPJs.

\section{Data Availability}

No data were used to support this study.

\section{Conflicts of Interest}

The authors declare that they have no conflicts of interest.

\section{Acknowledgments}

This work was supported by the National Natural Science Foundation of China (Grant nos. 11901584, 61876192, 61976228, and 62076106) and the Fundamental Research Funds for the Central Universities of South-Central University for Nationalities (Grant nos. CZY20013, CZY20014, CZT20020, CZT20022, KTZ20051, and YZZ19004).

\section{References}

[1] L. Arnold, Stochastic Differential Equations, Theory and Applications, John Wiley, New York, NY, USA, 1974.

[2] X. Mao, Stochastic Differential Equations and Applications, Horwood, Chichester, 2007.

[3] E. Allen, Modeling with Itô Stochastic Differential Equations, Springer, Dordrecht, Netherlands, 2007.
[4] D. J. Higham and P. E. Kloeden, "Numerical methods for nonlinear stochastic differential equations with jumps," Numerische Mathematik, vol. 101, no. 1, pp. 101-119, 2005.

[5] W. Mao, S. You, and X. Mao, "On the asymptotic stability and numerical analysis of solutions to nonlinear stochastic differential equations with jumps," Journal of Computational and Applied Mathematics, vol. 301, pp. 1-15, 2016.

[6] L.-S. Wang, C. Mei, and H. Xue, "The semi-implicit Euler method for stochastic differential delay equation with jumps," Applied Mathematics and Computation, vol. 192, no. 2, pp. 567-578, 2007.

[7] W. Mao, "Convergence of numerical solutions for variable delay differential equations driven by Poisson random jump measure," Applied Mathematics and Computation, vol. 212, pp. 409-417, 2009.

[8] X. Mao and C. Yuan, Stochastic Differential Equations with Markovian Switching, Imperial College Press, London, UK, 2006.

[9] P. E. Kloeden and E. Platen, Numerical Solution of Stochastic Differential Equations, Springer, Berlin, Germany, 1992.

[10] M. Hutzenthaler, A. Jentzen, and P. E. Kloeden, "Strong and weak divergence in finite time of Euler's method for stochastic differential equations with non-globally Lipschitz continuous coefficients," Proceedings of the Royal Society A: Mathematical, Physical and Engineering Sciences, vol. 467, no. 2130, pp. 1563-1576, 2010.

[11] D. J. Higham, X. Mao, and A. M. Stuart, "Strong convergence of Euler-type methods for nonlinear stochastic differential equations," SIAM Journal on Numerical Analysis, vol. 40, no. 3, pp. 1041-1063, 2002.

[12] G. N. Milstein, E. Platen, and H. Schurz, "Balanced implicit methods for stiff stochastic systems," SIAM Journal on $\mathrm{Nu}$ merical Analysis, vol. 35, no. 3, pp. 1010-1019, 1998.

[13] L. Szpruch, X. Mao, D. J. Higham, and J. Pan, "Numerical simulation of a strongly nonlinear Ait-Sahalia-type interest rate model," BIT Numerical Mathematics, vol. 51, no. 2, pp. 405-425, 2011.

[14] M. Hutzenthaler, A. Jentzen, and P. E. Kloeden, "Strong convergence of an explicit numerical method for SDEs with nonglobally Lipschitz continuous coefficients," Annals of Applied Probability, vol. 22, pp. 1611-1641, 2012.

[15] W. Liu and X. Mao, "Strong convergence of the stopped Euler-Maruyama method for nonlinear stochastic differential equations," Applied Mathematics and Computation, vol. 223, pp. 389-400, 2013.

[16] S. Sabanis, "Euler approximations with varying coefficients: the case of superlinearly growing diffusion coefficients," Annals of Applied Probability, vol. 26, pp. 2083-2105, 2016.

[17] X. Mao, "The truncated Euler-Maruyama method for stochastic differential equations," Journal of Computational and Applied Mathematics, vol. 290, pp. 370-384, 2015.

[18] X. Mao, "Convergence rates of the truncated Euler-Maruyama method for stochastic differential equations," Journal of Computational and Applied Mathematics, vol. 296, pp. 362375, 2016.

[19] S. Deng, W. Fei, W. Liu, and X. Mao, "The truncated EM method for stochastic differential equations with Poisson jumps," Journal of Computational and Applied Mathematics, vol. 355, pp. 232-257, 2019.

[20] Q. Guo, X. Mao, and R. Yue, "The truncated Euler-Maruyama method for stochastic differential delay equations," Numerical Algorithms, vol. 78, no. 2, pp. 599-624, 2018.

[21] L. Hu, X. Li, and X. Mao, "Convergence rate and stability of the truncated Euler-Maruyama method for stochastic 
differential equations," Journal of Computational and Applied Mathematics, vol. 337, pp. 274-289, 2018.

[22] G. Lan and F. Xia, "Strong convergence rates of modified truncated EM method for stochastic differential equations," Journal of Computational and Applied Mathematics, vol. 334, pp. 1-17, 2018.

[23] Q. Guo, W. Liu, X. Mao, and R. Yue, "The partially truncated Euler-Maruyama method and its stability and boundedness," Applied Numerical Mathematics, vol. 115, pp. 235-251, 2017.

[24] S. Gao, J. Hu, L. Tan, and C. Yuan, "Strong convergence rate of truncated Euler-Maruyama method for stochastic differential delay equations with Poisson jumps," Frontiers of Mathematics in China, vol. 16, no. 2, pp. 395-423, 2021.

[25] S. Gao and J. Hu, "Numerical method of highly nonlinear and nonautonomous neutral stochastic differential delay equations with Markovian switching," Advances in Difference Equations, vol. 2020, no. 1, 688 pages, 2020.

[26] A. Wu and Z. Zeng, "Global Mittag-Leffler stabilization of fractional-order memristive neural networks," IEEE Transactions on Neural Networks and Learning Systems, vol. 28, no. 1, pp. 206-217, 2017.

[27] A. Wu and Z. Zeng, "Output convergence of fuzzy neurodynamic system with piecewise constant argument of generalized type and time-varying input," IEEE Transactions on Systems, Man, and Cybernetics: Systems, vol. 46, no. 12, pp. 1689-1702, 2016.

[28] A. Wu and Z. Zeng, "Lagrange stability of memristive neural networks with discrete and distributed delays," IEEE Transactions on Neural Networks and Learning Systems, vol. 25, no. 4, pp. 690-703, 2014.

[29] A. Wu, H. Liu, and Z. Zeng, "Observer design and Ho performance for discrete-time uncertain fuzzy-logic systems," IEEE Transactions on Cybernetics, vol. 51, no. 5, pp. 23982408, 2021.

[30] A. Ailong Wu and Z. Zhigang Zeng, "Exponential stabilization of memristive neural networks with time delays," IEEE Transactions on Neural Networks and Learning Systems, vol. 23, no. 12, pp. 1919-1929, 2012.

[31] W. Liu, X. Mao, J. Tang, and Y. Wu, "Truncated EulerMaruyama method for classical and time-changed non-autonomous stochastic differential equations," Applied $\mathrm{Nu}$ merical Mathematics, vol. 153, pp. 66-81, 2020.

[32] L. Tan and C. Yuan, "Convergence rates of truncated thetaEM scheme for SDDEs," Scientia Sinica Mathematics (Chinese), vol. 50, pp. 137-154, 2020.

[33] L.-s. Wang and H. Xue, "Convergence of numerical solutions to stochastic differential delay equations with Poisson jump and Markovian switching," Applied Mathematics and Computation, vol. 188, no. 2, pp. 1161-1172, 2007.

[34] R. Li and Z. Chang, "Convergence of numerical solution to stochastic delay differential equation with Poisson jump and Markovian switching," Applied Mathematics and Computation, vol. 184, pp. 451-463, 2007.

[35] G. Wang and D. Li, "Algorithmic analysis of Euler-Maruyama scheme for stochastic differential delay equations with Markovian switching and Poisson jump, under non-Lipschitz condition," in Proceedings of the Fifth International Conference on Natural Computation, IEEE Computer Society, Tianjian, China, August 2009.

[36] H. Yang and F. Jiang, "Analytic approximation of the solutions of stochastic differential delay equations with Poisson jump and Markovian switching," Journal of Applied Mathematics, pp. 1409-1415, 2012.
[37] A. V. Skorohod, Asymptotic Methods in the Theory of Stochastic Differential Equations, American Mathematical Society, Providence, Rhode Island, USA, 1989.

[38] E. Zeidler, Nonlinear Functional Analysis and its Applications II, Springer-Verlag, New York, NY, USA, 1990.

[39] J. Bao, B. Böttcher, X. Mao, and C. Yuan, "Convergence rate of numerical solutions to SFDEs with jumps," Journal of Computational and Applied Mathematics, vol. 236, no. 2, pp. 119-131, 2011. 